\title{
Search for surface magnetic fields in Mira stars: first results on $\chi \mathrm{Cyg}$
}

\author{
Agnès Lèbre ${ }^{1}$, Michel Aurière ${ }^{2}$, Nicolas Fabas ${ }^{3}$, Denis Gillet ${ }^{4}$, Fabrice \\ Herpin $^{5}$, Pascal Petit ${ }^{2}$ and Renada Konstantinova-Antova ${ }^{6}$ \\ ${ }^{1}$ Laboratoire Univers et Particules de Montpellier, CNRS \& Université de Montpellier, France \\ email: Agnes.Lebre@univ-montp2.fr \\ ${ }^{2}$ IRAP, CNRS \& Université de Toulouse, Observatoire Midi-Pyrénées, Toulouse, France \\ ${ }^{3}$ Instituto de Astrofísica de Canarias \& Universidad de la Laguna, Tenerife, Spain \\ ${ }^{4}$ Observatoire de Haute Provence, OSU Pythéas, CNRS \& Aix-Marseille Université, France \\ ${ }^{5}$ Laboratoire d'Astronomie de Bordeaux, OASU, CNRS \& Université de Bordeaux, France \\ ${ }^{6}$ Institute of Astronomy and NAO, Bulgarian Academy of Sciences, Sofia, Bulgaria
}

\begin{abstract}
So far, surface magnetic fields have never been reported on Mira stars, while observational facilities allowing detection and measurement of weak surface fields through the Zeeman effect have become available. Then, in order to complete the knowledge of the magnetic field and of its influence during the transition from Asymptotic Giant Branch (AGB) to Planetary Nebulae $(\mathrm{PN})$ stages, we have undertaken a search for magnetic fields at the surface of Miras. We present the first spectropolarimetric observations (performed with the Narval instrument at Télescope Bernard Lyot-TBL, Pic du Midi, France) of the S-type Mira star $\chi$ Cyg. We have detected a polarimetric signal in the Stokes V spectra and we have established its Zeeman origin. We claim that it is likely to be related to a weak magnetic field present at the photospheric level and in the lower part of the stellar atmosphere. The origin of this magnetic field is discussed in the framework of shock waves periodically propagating throughout the atmosphere of a Mira.
\end{abstract}

Keywords. Stars: AGB and post-AGB, stars: atmospheres, Magnetic fields, shock waves

\section{Introduction}

Miras are cool and evolved pulsating stars, belonging to the AGB, the key evolutionary stage of an intermediate mass star before its transition toward the PN stage. These evolved stars are known to be among the main recycling agents of the interstellar medium as they undergo a prodigious mass loss supposedly mainly driven by radiation pressure combined to other factors (magnetism, pulsation) whose respective contributions are not yet fully identified. For Mira stars a radiative shock wave triggered by the pulsation mechanism and propagating periodically throughout the extended stellar atmosphere may help to form and to enrich a circumstellar envelope (hereafter CSE). The morphology of the CSE of an AGB star is known to severely change during its quick transition toward PN (Sahai \& Trauger, 1998). The classical or generalized Interacting Stellar Winds model (Kwok, 2000) tries to explain this shaping, but does not fully address the origin of the wind as it fails in reproducing the observed complex structures (such as jets or ansae). Then binarity and/or magnetic fields have often been invoked in order to rule the mass loss geometry and to shape PN's morphology (Soker, 2006; Blackman, 2009). The presence of a weak magnetic field at the surface of Miras is also expected from theoretical considerations : the ejection of massive winds by an AGB could be triggered by a magnetic activity in the degenerated core, leading to a toroidal field configuration of a few $10 \mathrm{G}$ at the stellar surface (Pascoli, 1997). 
Observational evidences for magnetic fields around PN and their AGB and post-AGB progenitors have been recently established (Sabin et al., 2007; Vlemmings, 2011). Magnetic fields have been detected and measured within the CSE of AGBs through the polarization from the maser emission of several molecules $\left(\mathrm{OH}, \mathrm{H}_{2} \mathrm{O}\right.$ and $\left.\mathrm{SiO}\right)$, located at different distances from the central star. The current status on magnetic field, $B_{/ /}$ (mean value of the intensity of the magnetic field along the line of sight) throughout the CSE of an AGB star is the following (with one stellar radius $\mathrm{R}_{*} \sim 1 \mathrm{au}$ ):

- $B_{/ /} \sim 5-20 \mathrm{mG}$, from OH masers, at 1000-10000 au (Rudnitski et al., 2010),

- $B / / \sim$ a few $100 \mathrm{mG}$, from $\mathrm{H}_{2} \mathrm{O}$ masers, at a few $100 \mathrm{au}$ (Leal-Ferreira et al., 2013)

- $B_{/ /} \sim$ a few $\mathrm{G}$ (mean value = 3.5 G), from SiO masers, at 5-10 au (Herpin et al., 2006), the innermost detections of magnetic fields within the environment of Miras.

Combining those values (and few detections reported in the very outer regions of the CSE of carbon-rich AGBs obtained from the Zeeman effect in CN line emission, Herpin et al., 2009), Vlemmings (2011) has presented the behavior of magnetic field strength within the environment of AGBs. Throughout the CSE of an AGB, the magnetic field strength presents a clear decrease along $\mathrm{r}$ (distance to the central object) favoring a $1 / \mathrm{r}$ variation law. Extrapolating this law, for a toroidal field configuration, one can expect a magnetic field strength of the order of few $G$ at a Mira's photosphere. Its detection and characterization are hence possible with a spectropolarimeter like Narval at TBL, as weak fields $\left(B_{/ /}\right.$between 1 to $10 \mathrm{G}$ ) have been detected at the surface of cool giants and AGBs with spectral types from K0III to M5III (Aurière et al., 2009; KonstantinovaAntova et al., 2010; Konstantinova-Antova et al., contribution in this volume) and also at the surface of the red supergiant (RSG) Betelgeuse (Aurière et al., 2010).

\section{Narval observations of $\chi$ Cyg : detection of a Stokes V signature}

The S type bright Mira star $\chi$ Cyg has a pulsation period of about 408 days. Herpin et al., (2006) have detected a magnetic field (from SiO maser emission) in the inner part of its CSE, reporting a mean value of $5 \mathrm{G}$ at a few stellar radii.

In March 2012, we have performed spectropolarimetric observations of $\chi$ Cyg, observed around its maximum light. We have collected 174 Stokes V sequences (circular polarization data) with Narval at TBL. We have performed a Least Squares Deconvolution (LSD) analysis (Donati et al., 1997) onto the combination of these V spectra, using a numeric mask dedicated to a cool and evolved star $\left(T_{\text {eff }}=3500 \mathrm{~K}, \log g=0.5\right.$, microturbulence $\xi=2 \mathrm{~km} . \mathrm{s}^{-1}$, solar abondances) and involving $\sim 14000$ metallic lines (distributed from 380 to $1000 \mathrm{~nm}$ ) with atomic parameters and Landé factors taken from the VALD database. In Fig. 1, we report the detection of a statistically significant signal in the averaged LSD Stokes V profile (with a $10^{-5}$ amplitude level). The Stokes I profile (unpolarized spectrum) is also presented as well as the Null profile (N) diagnosis. The Null profile remains flat within the line, confirming the definite detection in Stokes V. The LSD Stokes I profile presents the typical doubling of metallic lines (Schwarzschild, 1952) due to the presence of a radiative shock wave imprinting complex ballistic motions in the lower part of the stellar atmosphere (Gillet et al., 1983).

\section{Physical origin of the detected Stokes V signature}

A magnetic origin. We have performed several tests on the combination of the 174 Stokes V spectra, in order to confirm its stellar origin, and we have found that indeed spurious or instrumental effects (crosstalk) do not contribute to its occurence. Moreover, we have also performed LSD analyses using two different numeric masks (both issued 
from the initial one) and gathering atomic lines with high or low Landé factor values (cut off value $=1.2$ ). In Fig. 2, we present the resulting LSD Stokes V profiles of these analyses, revealing a definite detection when using the numeric mask gathering lines with high Landé factor values. The clear Stokes V signature obtained with this sub mask presents the same structure, amplitude and location than the one obtained when using the initial mask (Fig. 1). This behavior confirms a magnetic Zeeman effect origin for the $\mathrm{V}$ signal detected on $\chi \mathrm{Cyg}$ and it represents the first detection - at the stellar surface of a Mira star - of a weak magnetic field. We have thus computed the longitudinal magnetic field $\left(B_{/ /}\right)$using the first-order moment method (Donati et al., 1997) adapted to the obtained mean LSD I and V profiles : $\mathrm{B}_{/ /}=-0.25 \pm 0.40 \mathrm{G}$.

A link with the periodic radiative shock wave. However, the estimation of $\mathrm{B}_{/ /}$has been made using the complete width of the Stokes I profile, and it could thus be considered as a lower limit. Indeed the Stokes V profile appears to be associated to the blue component of the LSD Stokes I profile (Fig. 1), i.e., to the material which is driven outward by the radiative shock wave. This suggests that the shock may have an impact onto a surface stellar magnetism, likely producing a compressive amplification of a very weak field. Thus we have performed a direct scaling of the Stokes V signal and the blue component of the I profile of $\chi \mathrm{Cyg}$, to the classical Zeeman profile obtained in the (non-pulsating) K0III star Pollux (Aurière et al., 2009). Then the Stokes V signal we report for $\chi$ Cyg would correspond to the detection of a surface magnetic field with $\mathrm{B}_{/ /}$of a few Gauss (2-3 G). This result is in good agreement with the measurements performed in the inner part of the CSE of $\chi$ Cyg. It also favors a decreasing law throughout the stellar environment in $1 / \mathrm{r}$ (associated to a toroidal field configuration). As already reported for the RSG Betelgeuse (Dorch, 2004 ; Petit et al., 2013), we suggest that a local dynamo powered by the convection and/or the atmospheric dynamics may be a likely explanation for the surface magnetism we have detected in $\chi \mathrm{Cyg}$, the propagation of the radiative shock wave probably inducing a compressive amplification on a weak surface field.

Further perspectives for this work rely on monitoring Miras around their maximum light and over a large part of their pulsation cycle, so as to precise the relation between

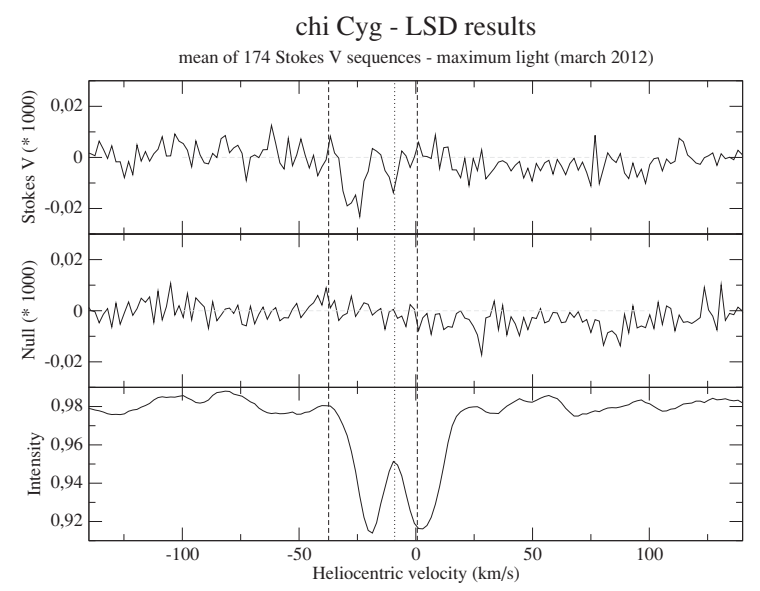

Figure 1. LSD profiles of $\chi \mathrm{Cyg}$ (in heliocentric velocity) from Narval observations collected in March 2012 (average of $174 \mathrm{~V}$ sequences). Bottom: Stokes I (unpolarized spectrum). Middle: The Null profile (extended by a factor of 1000 ). Top: Stokes V profile (extended by a factor of $1000)$. The vertical dashed lines delimitate the blue line component of the Stokes I profile. 
chi Cyg (maximum light 2012)

LSD results (using mask with low/high Lande factor lines)

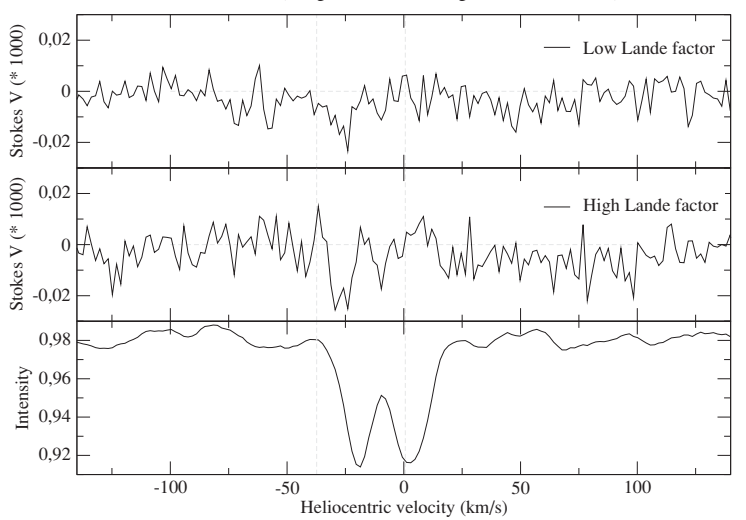

Figure 2. LSD Stokes I and V profiles of $\chi$ Cyg (in heliocentric velocity). Bottom: LSD Stokes I profile (see Fig. 1). Middle: LSD Stokes V profile obtained with a numeric mask gathering atomic lines with high Landé factor values (mean value $=0.9$ ). Top: LSD Stokes V profile obtained with a numeric mask gathering atomic lines with low Landé factor values (mean value $=1.5$ ).

the atmospheric shock wave and the stellar magnetism. The sub gauss level detection is required for such observations, and in a next future, SPIRou at CFHT will be very useful for the detection and the investigation of surface magnetic fields in cool and evolved stars.

\section{References}

Aurière, M., Wade, G., Konstantinova-Antova, R., et al., 2009, A\&A 504, 231

Aurière, M., Donati, J. F., \& Konstantinova-Antova, R., 2010, A $\mathcal{A} A$ 516, L2

Blackman, E. G., 2009, in "Cosmic Magnetic Fields", eds. Strassmeier et al., IAU Symp. 259, 35

Donati, J. F., Semel, M., Carter, B., Reed, D., \& Collier Cameron, A., 1997, MNRAS 291, 658

Dorch, S. B. F., 2004, A\&\&A, 423, 1101

Gillet, D., Maurice, E., \& Baade, D., 1983, A\& $A$ 128, 384

Herpin, F., Baudry, A., Thum, C., Morris, D., \& Wiesemeyer, H., 2006, A\&A 450; 667

Herpin, F., Baudry, A., Josselin, E., Thum, C., \& Wiesemeyer, H., 2009, in "Cosmic Magnetic Fields", eds. Strassmeier et al., IAU Symp. 259, 47

Konstantinova-Antova, R., Aurière, M., Charbonnel, C., et al., 2010, A\&A, 524, A57

Kwok, S., 2000, ASPC 199, 9

Leal-Ferreira, M. L., Vlemmings, W. H. T., Kemball, A., \& Amiri, N., 2013, A\& A, 554, A134

Pascoli, G., 1997, ApJ 489, 946

Petit, P., Aurière, M. , Konstantinova-Antova, R., Morgenthaler, M., et al., 2013 LNP 857, 231

Rudnitski, G. M., Paschenko, M. I., \& Colom, P., 2010, Astronomy Reports, Vol. 54, p. 400

Sabin, L., Zijlstra, A. A., \& Greaves, J. S., 2007, MNRAS 376, 378

Sahai, R. \& Trauger, J. T., 1998, AJ 116, 1357

Schwarzschild, M., 1952, Transact. IAU VIII, ed. P. Oosterhoff, Cambridge Univ. Press, p 811

Soker, N., 2006, PASP 118, 260

Vlemmings, W. H. T., 2011, Proceedings of AsPNe Conf., eds. A. Zijlstra et al., p 91 